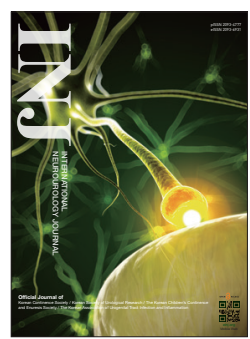

\title{
Animal Modelling of Interstitial Cystitis/Bladder Pain Syndrome
}

\author{
Lori Birder ${ }^{1,2}$, Karl-Erik Andersson ${ }^{3,4}$ \\ Departments of ${ }^{1}$ Medicine and ${ }^{2}$ Pharmacology and Chemical Biology, University of Pittsburgh School of Medicine, Pittsburgh, PA, USA \\ ${ }^{3}$ Institute for Regenerative Medicine, Wake Forest University School of Medicine, Winston Salem, NC, USA \\ ${ }^{4}$ Institute of Laboratory Medicine, Lund University, Lund, Sweden
}

\begin{abstract}
The etiology of interstitial cystitis/bladder pain syndrome (IC/BPS) remains elusive and may involve multiple causes. To better understand its pathophysiology, many efforts have been made to create IC/BPS models. Most existing models of IC/BPS strive to recreate bladder-related features by applying noxious intravesical or systemic stimuli to healthy animals. These models are useful to help understand various mechanisms; however, they are limited to demonstrating how the bladder and nervous system respond to noxious stimuli, and are not representative of the complex interactions and pathophysiology of IC/BPS. To study the various factors that may be relevant for IC/BPS, at least 3 different types of animal models are commonly used: (1) bladder-centric models, (2) models with complex mechanisms, and (3) psychological and physical stressors/natural disease models. It is obvious that all aspects of the human disease cannot be mimicked by a single model. It may be the case that several models, each contributing to a piece of the puzzle, are required to recreate a reasonable picture of the pathophysiology and time course of the disease(s) diagnosed as IC/BPS, and thus to identify reasonable targets for treatment.
\end{abstract}

Keywords: Interstitial cystitis; Bladder pain syndrome; Animal models; Inflammation; Mucosa; Stress

- Conflict of Interest: LB and KEA, deputy Editors-in-Chief of the INJ, are the authors of this article. However, they played no role whatsoever in the editorial evaluation of this article or the decision to publish it. No potential conflict of interest relevant to this article was reported.

\section{INTRODUCTION}

Interstitial cystitis/bladder pain syndrome (IC/BPS) is a chronic pain disorder involving symptoms of urinary frequency, urgen$\mathrm{cy}$, and pain. This condition is difficult to diagnose, with some describing 2 separate disorders: 'interstitial cystitis' as a chronic inflammatory disorder, and 'bladder pain syndrome,' which often lacks an inflammatory component [1]. Regardless, the overall etiology of IC/BPS remains elusive and may involve multiple causes. To better understand its pathophysiology, both subchronic and chronic cystitis animal models have been introduced, and many attempts have been made to create IC/BPS models. Animal models of bladder inflammation have been ex- tensively discussed previously (e.g., [2]). The present review is focused specifically on some models commonly used to mimic the features of findings in IC/BPS patients. These models can be broadly categorized as (1) bladder-centric models, (2) models with complex mechanisms, and (3) psychological and physical stressors/natural models.

\section{BLADDER-CENTRIC MODELS}

These models (most often rat and mouse) seem to mimic circumstances in which there is a toxic substance in the urine. These models can include the instillation of irritants such as hydrochloric or acetic acid [3,4], acetone [5], acrolein (the active
Corresponding author: Lori Birder (iD http://orcid.org/0000-0002-3393-2256 University of Pittsburgh School of Medicine, A 1217 Scaife Hall, 3550 Terrace Street, Pittsburgh, PA 15261, USA

E-mail: lbirder@pitt.edu / Tel: +1-412-383-7368 / Fax: +1-412-648-7197

Submitted: November 18, 2017 / Accepted after revision: January 3, 2018
This is an Open Access article distributed under the terms of the Creative Commons Attribution Non-Commercial License (http://creativecommons.org/licenses/by-nc/4.0/) which permits unrestricted non-commercial use, distribution, and reproduction in any medium, provided the original work is properly cited. 
metabolite of cyclophosphamide) [6,7], xylene [8], mustard oil, croton oil, turpentine [9], protamine sulfate [10], lipopolysaccharide [11,12], or cyclophosphamide (CYP; [2]). They can also can include the altered expression of urothelial targets (i.e., claudins [13], antiproliferative factors [14], and uroplakin II [4]).

A commonly used bladder-centric model of acute bladder pain is the rodent CYP-induced cystitis model, induced with a single intraperitoneal injection of CYP. This model exhibits many typical features of BPS within 24 hours after injection, such as pain-related behaviours, bladder overactivity, and activation of inflammation. The bladder shows oedema, massive inflammatory cell infiltration, haemorrhages, and mucosal destruction, which make this model more relevant as an acute haemorrhagic cystitis model than as a model of IC/BPS. A chronic model of milder bladder pain, induced by multiple systemic injections of lower doses of CYP and eliciting chronic inflammation without pronounced behavioural changes, has been described in rats [15-17] and mice [18,19].

The CYP models have been used to study the pathological changes in bladder neuronal pathways associated with chronic inflammation. The molecular mechanisms underlying the pathological changes characteristic of this model have been extensively investigated by many researchers, such as Vizzard and collaborators [20-25]. Nonetheless, the translational importance of these findings remains to be elucidated. In rats, even low doses of CYP induce severe inflammation and damage in bladder tissue $[17,26]$, which is atypical for nonulcerative BPS patients.

Mice, unlike rats, appear to be more resistant to systemic CYP treatment. Boudes et al. [18] demonstrated that chronic low-dose administration of CYP to C57Bl6 male mice caused detrusor overactivity, increased urinary frequency, and hyperalgesia of the lower abdominal area without impairment of the physiological state, overt damage to bladder tissue, or changes in body temperature and weight. In this chronic model, oedema and lymphocyte invasion in the bladder lamina propria were associated with hyperplasia of the urothelium rather than denudation, erosions, or thinning. Using a similar protocol, Lai et al. [27] showed the development of bladder hyperalgesia in female C57 mice by measuring the abdominal visceromotor response to bladder distension. Altogether, these data demonstrate that a mouse model of chronic CYP-induced bladder inflammation and pain can be considered as a promising extension of preclinical BPS research. Golubeva et al. [19] showed that mice subjected to repetitive systemic injections of CYP de- veloped a mild inflammatory response in bladder tissue characterized by oedema of the lamina propria, a moderate increase in proinflammatory cytokine gene expression, and mastocytosis. No signs of massive inflammatory infiltrate, tissue haemorrhages, mucosal ulcerations, or urothelium loss were observed. Instead, CYP treatment induced urothelium hyperplasia, accompanied by the activation of proliferative signalling cascades, and a decrease in the expression of urothelium-specific markers.

Bladder-centric models have clear limitations and are focused on what may be occurring in the bladder. Acute CYP administration causes haemorrhagic cystitis, which is typical for neither IC nor BPS. Repeated administration of low-dose CYP induces "chronic" cystitis, but these models are more appropriately considered to be repetitive acute cystitis models, at least in rats. Chronic CYP treatment of mice causes urothelial hyperplasia, which is not a characteristic of IC/BPS. Agents such as protamine are nonselective, and as such can disrupt the cytoplasmic membrane, resulting in cell lysis [28]. This can result in corresponding damage to multiple cell types extending beyond the urothelial layer (unpublished observations), so they should not be considered to be appropriate agents for modelling IC/ BPS. To date, no available model has attempted to mimic the specific epidemiologic findings of IC/BPS patients or has accounted for the known effect of sustained adult stress on symptom severity. Even if bladder-centric animal models cannot be considered to model the human disease, they are important for elucidating pathological changes and underlying mechanisms in bladder structures associated with chronic inflammation induced in various ways.

\section{MODELS WITH COMPLEX MECHANISMS}

Models with complex mechanisms are based on the assumption that the causes for the alterations in the bladder occur elsewhere, such as via activation of the central nervous system (CNS). These models include injection of pseudorabies virus (PRV) in the tail, leading to bladder changes via the activation of CNS circuitry and various comorbid disorders (e.g., instillation of TNBS (2,4,6-trinitrobenzenesulfonic acid) into the colon, autoimmune models, chronic pelvic pain, and vulvodynia) [29-33].

PRV infection in mice induces a form of neurogenic cystitis associated with bladder lamina propria mast cell accumulation and pelvic pain. Rudick et al. [34] induced neurogenic cystitis by the injection of Bartha's strain of PRV into the abductor cau- 
dalis dorsalis tail base muscle of female C57BL/6 mice. They used this model to demonstrate that the PRV-induced pelvic pain could be reduced in animals treated with antagonists of neurokinin receptor 1 and histamine receptors. More recent studies by this group using PRV-induced neurogenic cystitis in mice have identified acyloxyacyl hydrolase (Aoah) as a novel locus that is associated with pelvic allodynia and modulates pelvic pain [35]. The group showed that Aoah-deficient mice exhibit features of IC with elevated levels of certain biomarkers (vascular endothelial growth factor) that are also highly expressed in IC patients. Sadler et al. [36] described a mouse model of urinary bladder distension in which compressed air at a specific pressure is delivered to the bladder of a lightly anesthetized animal over a set period of time. Electrical activity in the superior oblique abdominal muscles is recorded as the visceromotor response, which is a reliable and reproducible measure of nociception. Wang et al. [37] used a transgenic autoimmune cystitis model that reproduces many clinical correlates of IC/BPS to investigate the role of mast cells in cystitis. They concluded that their results provided direct evidence for the role of mast cells in cystitis-associated lower urinary tract dysfunction, and that they supported the use of mast cell inhibitors to treat certain forms of IC/BPS.

These models may provide interesting insights in mechanisms that can contribute to cystitis/bladder dysfunction, but their translational value for IC/BPS is unclear.

\section{PSYCHOLOGICAL AND PHYSICAL STRESSORS/ NATURAL MODELS}

This category includes various stress models (water avoidance stress [WAS], restraint stress), a feline IC model, and models involving the injection of norepinephrine and the manipulation of environmental temperature or light. While animals cannot self-report whether they can feel pain, their behavior in response to various noxious or otherwise irritative stimuli can be observed and scored. Recently, studies have shown that other factors, including various types of psychological and physical forms of stress, can produce either analgesia or hyperalgesia in a number of settings. Even differences in ambient temperature and social factors can alter measures in pain studies. Psychosocial stress can have a significant impact on several comorbid and other diseases, including fibromyalgia, skin diseases, and even asthma and irritable bowel syndrome (IBS). A wealth of research has revealed that stress can significantly alter commu- nication in the (skin, bladder, and gut)-brain axis and that targeting these signaling mechanisms may impact health and disease [38-40]. In addition, IC/BPS patients exhibit increased functional brain activation with a full bladder in brain regions known to participate in sensory perception and pain. In this regard, there is evidence that chronic WAS in rats predisposed to anxiety can result in a number of clinical and functional features similar to those observed in humans diagnosed with IC/ BPS. Studies have shown that chronic psychological stress induced urinary frequency, sustained bladder hyperalgesia, tactile hindpaw allodynia, and suprapubic hyperalgesia $[41,42]$. This model has also revealed increased engagement of portions of the micturition circuit responsive to urgency [43]. The WAS model has also been shown to exhibit alterations in the gastrointestinal (GI) tract as a model for IBS, which may be a comorbid condition associated with both lower urinary tract symptoms (LUTS) and IC/BPS.

While the sequence of events that link psychological stress to functional pain syndromes such as IC/BPS has not been clarified, it is thought that activation of the sympathetic division of the autonomic nervous system may play a key role. The involvement of the sympathetic system has been reported in a number of chronic painful conditions, including complex regional pain syndrome and fibromyalgia. For the latter, studies have revealed that the sympatho-adrenal stress axis can play a role not only in the induction, but also the maintenance of mechanical hyperalgesia. Adrenergic stimulation has been shown to mediate hypersensitivity of the pelvic viscera, both in terms of long-lasting effects on bladder function and in terms of colorectal distension. Thus, chronic stress results in enduring changes in the nervous system that alter sympathetic nerve activity, which may be a mechanism by which stress enhances the severity and duration of pain symptoms.

The feline IC model is a naturally occurring model of IC. No other model reproduces as many features of IC as this model, including comorbidities with other unexplained clinical conditions and increased norepinephrine content $[44,45]$. Chronic WAS in rodents is considered to have high construct and face validity for bladder hypersensitivity syndromes, such as IC/ BPS. It is characterized by urinary frequency and bladder hyperalgesia and heightened stress responsiveness $[43,46]$. The bladder of WAS animals shows inflammatory cell infiltration in the mucosa and a patchy decrease in the urothelial surface due to a loss of the superficial umbrella cells. Charrua et al. [47] used a model where phenylephrine was given subcutaneously 
daily for 14 days. Phenylephrine administration increased visceral pain, spinal Fos expression, bladder reflex activity, urinary spotting, and the number of expelled fecal pellets. The mucosa showed urothelial thinning, and mastocytosis was present in the suburothelium. The sympathetic nerve density and urinary noradrenaline levels increased. Induced pain in this model was reversed by silodosin [48].

The feline IC model, even if it is the most similar to human disease, is limited by the scarce availability of animals, and does not seem to be currently used. The WAS models and the phenylephrine model currently seem to be the most interesting, because they demonstrate not only bladder changes, but also systemic alterations (e.g., central sensitization) that may be found in IC/BPS patients. In addition, a National Institutes of Health initiative, the Multi-Disciplinary Approach to the Study of Chronic Pelvic Pain research network, has evaluated animal models to study urological chronic pelvic pain syndrome $[49,50]$. The WAS model reflects the stress variability of the human condition, and exhibits many of the key symptoms that occur in patients.

\section{APPLICATION OF ANIMAL MODELS FOR THE STUDY OF IC/BPS}

There are many caveats and concerns to consider when assessing the translational impact of the various models that have been claimed to mirror IC/BPS. An important question is: which aspects of a complex human disease such as IC/BPS can be modeled in animals? No animal model can be expected to reproduce all the various symptoms experienced by humans, and it is obvious that all aspects of the disease cannot be mimicked by a single model. Furthermore, it is widely accepted that IC and BPS are different disorders with similar symptoms, but with different bladder pathologies. Since it is not established whether IC/BPS is a bladder disease that sometimes has systemic manifestations or a systemic disorder with bladder manifestations, the bladder-centric models have obvious limitations. For example, the effects of intravesical instillation of an irritant or immune stimulant, which have been investigated in a number of species (guinea pigs, rodents, rabbits, and cats), exhibit a great deal of variability in terms of the type and concentration of irritant, the volume instilled (which affects the urothelial barrier, amongst other considerations) and the time of exposure. Each change in a parameter can alter the amount and duration of the effect on an epithelial surface, thereby producing differ- ing results. A noxious agent can affect multiple epithelial surfaces, so the resulting changes may be nonspecific effects due to injury. Additionally, histologic changes may occur, but not functional changes. Multiple types of experiments (bladder strips, cystometrograms, etc.) may yield incremental pieces of evidence, but be too disparate for any logical conclusion relevant to IC/BPS. The time course and resolution of any form of acute inflammation differs from that in most patients (in whom inflammation does not resolve). Thus, the net effect for most (not all) models using animals, while useful, may not mirror patient symptoms, which typically are chronic and wax and wane. A chronic model capable of mirroring patients who show both peripheral and central sensitization can be expected to involve several systems/mediators. The use of biomarkers, especially in human tissue, is fraught with problems given the lack of standardization of where biopsies are taken, the depth of the biopsies, and so on. The same problems are relevant for animal tissue (or blood/urine samples). Additionally, how and when the urine is collected (full vs. empty bladder) can affect the amount and type of mediators released for any measurement.

What can then be studied in animal models attempting to reproduce characteristics of IC/BPS in humans? Is it fruitful to study inflammatory changes in the bladder, guided by the findings of human bladder biopsies? Elbadawi [51] stated that "no specific or diagnostic light microscopic pathologic features are provided by either routine histopathology or immunohistochemistry." Despite this, Tomaszewski et al. [52], using these techniques, reported 4 statistically significant pathologic changes in IC patients: (1) the mast cell count in lamina propria with tryptase staining; (2) complete loss of the urothelium; (3) granulation tissue in the lamina propria; and (4) vascular density in the lamina propria with factor VIII staining. The percentage of the mucosa denuded of urothelium and the percentage of suburothelial haemorrhage remained closely associated with pain in a multivariable predictive model. These results suggest that histopathologic features can play an important role in the predictive modelling of IC symptoms, a view supported by Leiby et al. [53], who suggested that the use of biopsy data appeared to be a powerful, parenchyma-oriented approach to subgroup assignment. Even if Elbadawi [51] questioned the importance of factors such as mast cells from a diagnostic point of view, he demonstrated by electron microscopy the presence of mast cells, activated by piecemeal degranulation, in close proximity to intrinsic nerves, particularly in the suburothelium. Sant et al. [54] found that detrusor mastocytosis occurred in both classic and 
nonulcerative IC. In classic IC, mast cells were 6 to 8 fold more common in the detrusor compared with controls and 2 to 3 fold higher than in nonulcerative IC. Logadottir et al. [55] stated that in patients with nonulcerative IC, scarcely any inflammation was found in the bladder mucosa, and mast cells were not even noted in most cases. Most investigators now favour the view that IC and BPS are distinct entities [55,56], with IC being a disease and BPS a syndrome. Only about $12 \%$ of IC/ BPS patients suffer from classic (ESSIC 3C) IC [53]. Does this mean that bladder-centric models are of value only for studies of classic IC, and that if the histopathological changes in classic IC patients can be demonstrated in an animal model, such a model has a predictive value when testing a therapeutic intervention? Which models have predictive value for patients with BPS? Are complex-mechanism or 'natural' models preferable? As discussed above, WAS models in rats predisposed to anxiety can result in a number of clinical and functional features similar to those observed in humans diagnosed with IC/BPS. Since this model has also been shown to exhibit alterations in the GI tract as a model for IBS, which may be a comorbid condition associated with both LUTS and IC/BPS, it may yield new insights into the presumed systemic pathophysiology of these disorders.

\section{SUMMARY AND CONCLUSIONS}

The etiology of IC/BPS is unknown, but may involve multiple causes. It is now generally accepted that IC and BPS may be 2 different entities, so each disorder may require distinct animal models. However, 20 years ago Elbadawi [51] wrote: "As it now stands, there is no natural or induced animal model that duplicates IC as it occurs in humans." This remains true today. The limited success in translating the vast amount of basic scientific data obtained using animal models into effective and safe treatments for a variety of pain conditions may be due in large part to the complexities of the human condition itself. Thus, no animal model can be expected to reproduce all the various symptoms experienced by humans. Most available animal models strive to reproduce morphological and functional changes found in the bladders of patients; as such, they are bladder-centric, and may be useful for studies of, for instance, inflammatory pathways in IC patients. More complex models are needed for mimicking the symptoms and systemic changes found in BPS patients. All aspects of the human disease cannot be mimicked by a single animal model, and it may be the case that that several models, each contributing to a piece of the puzzle, are required to create a reasonable picture of the pathophysiology and time course of the disease(s) diagnosed as IC or BPS, and thus to identify reasonable targets for treatment.

\section{REFERENCES}

1. Payne CK. The Empereror Has No Clothes. Lecture AUA, 2017 [Internet]. Berkeley (CA): UroToday; c2002-2017 [cited 2007 Nov 27] Available from: https://www.urotoday.com/video-lectures/bpsic/video/788-embedded-media2017-07-05-16-32-47.html.

2. Bjorling DE, Wang ZY, Bushman W. Models of inflammation of the lower urinary tract. Neurourol Urodyn 2011;30:673-82.

3. Kirimoto T, Nakano K, Irimura K, Hayashi Y, Matsuura N, Kiniwa $\mathrm{M}$, et al. Beneficial effects of suplatast tosilate (IPD-1151T) in a rat cystitis model induced by intravesical hydrochloric acid. BJU Int 2007;100:935-9.

4. Song PH, Chun SY, Chung JW, Kim YY, Lee HJ, Lee JN, et al. Comparison of 5 different rat models to establish a standard animal model for research into interstitial cystitis. Int Neurourol J 2017;21:16370.

5. Kato K, Kitada S, Longhurst PA, Wein AJ, Levin RM. Time-course of alterations of bladder function following acetone-induced cystitis. J Urol 1990;144:1272-6.

6. Sakata T, Smith RA, Garland EM, Cohen SM. Rat urinary bladder epithelial lesions induced by acrolein. J Environ Pathol Toxicol Oncol 1989;9:159-69.

7. Bjorling DE, Elkahwaji JE, Bushman W, Janda LM, Boldon K, Hopkins WJ, et al. Acute acrolein-induced cystitis in mice. BJU Int 2007;99:1523-9.

8. Abelli L, Conte B, Somma V, Maggi CA, Giuliani S, Meli A. A method for studying pain arising from the urinary bladder in conscious, freely-moving rats. J Urol 1989;141:148-51.

9. McMahon SB, Abel C. A model for the study of visceral pain states: chronic inflammation of the chronic decerebrate rat urinary bladder by irritant chemicals. Pain 1987;28:109-27.

10. Stein PC, Pham H, Ito T, Parsons CL. Bladder injury model induced in rats by exposure to protamine sulfate followed by bacterial endotoxin. J Urol 1996;155:1133-8.

11. Jerde TJ, Bjorling DE, Steinberg H, Warner T, Saban R. Determination of mouse bladder inflammatory response to E. coli lipopolysaccharide. Urol Res 2000;28:269-73.

12. Tambaro S, Casu MA, Mastinu A, Lazzari P. Evaluation of selective cannabinoid $\mathrm{CB}(1)$ and $\mathrm{CB}(2)$ receptor agonists in a mouse model of lipopolysaccharide-induced interstitial cystitis. Eur J Pharmacol 
2014;729:67-74.

13. Montalbetti N, Rued AC, Clayton DR, Ruiz WG, Bastacky SI, Prakasam HS, et al. Increased urothelial paracellular transport promotes cystitis. Am J Physiol Renal Physiol 2015;309:F1070-81.

14. Keay S, Leitzell S, Ochrzcin A, Clements G, Zhan M, Johnson D. A mouse model for interstitial cystitis/painful bladder syndrome based on APF inhibition of bladder epithelial repair: a pilot study. BMC Urol 2012;12:17.

15. Vizzard MA, Erdman SL, de Groat WC. Increased expression of neuronal nitric oxide synthase in bladder afferent pathways following chronic bladder irritation. J Comp Neurol 1996;370:191-202.

16. Hu VY, Malley S, Dattilio A, Folsom JB, Zvara P, Vizzard MA. COX-2 and prostanoid expression in micturition pathways after cyclophosphamide-induced cystitis in the rat. Am J Physiol Regul Integr Comp Physiol 2003;284:R574-85.

17. Juszczak K, Gil K, Wyczolkowski M, Thor PJ. Functional, histological structure and mastocytes alterations in rat urinary bladders following acute and [corrected] chronic cyclophosphamide treatment. J Physiol Pharmacol 2010;61:477-82.

18. Boudes M, Uvin P, Kerselaers S, Vennekens R, Voets T, De Ridder D. Functional characterization of a chronic cyclophosphamide-induced overactive bladder model in mice. Neurourol Urodyn 2011; 30:1659-65.

19. Golubeva AV, Zhdanov AV, Mallel G, Dinan TG, Cryan JF. The mouse cyclophosphamide model of bladder pain syndrome: tissue characterization, immune profiling, and relationship to metabotropic glutamate receptors. Physiol Rep 2014;2:e00260.

20. Yoshimura N, de Groat WC. Increased excitability of afferent neurons innervating rat urinary bladder after chronic bladder inflammation. J Neurosci 1999;19:4644-53.

21. Ishigooka M, Zermann DH, Doggweiler R, Schmidt RA, Hashimoto T, Nakada T, et al. Spinal NK1 receptor is upregulated after chronic bladder irritation. Pain 2001;93:43-50.

22. LaBerge J, Malley SE, Zvarova K, Vizzard MA. Expression of corticotropin-releasing factor and CRF receptors in micturition pathways after cyclophosphamide-induced cystitis. Am J Physiol Regul Integr Comp Physiol 2006;291:R692-703.

23. Arms L, Girard BM, Vizzard MA. Expression and function of CXCL12/CXCR4 in rat urinary bladder with cyclophosphamideinduced cystitis. Am J Physiol Renal Physiol 2010;298:F589-600.

24. Girard BM, Cheppudira BP, Malley SE, Schutz KC, May V, Vizzard MA. Increased expression of interleukin- 6 family members and receptors in urinary bladder with cyclophosphamide-induced bladder inflammation in female rats. Front Neurosci 2011;5:20.

25. Dugan C, Malley S, Arms L, May V, Vizzard MA. Role of c-Jun N- terminal kinase (JNK) activation in micturition reflexes in cyclophosphamide (CYP)-induced cystitis in female rats. J Mol Neurosci 2014;54:360-9.

26. Malley SE, Vizzard MA. Changes in urinary bladder cytokine mRNA and protein after cyclophosphamide-induced cystitis. Physiol Genomics 2002;9:5-13.

27. Lai HH, Qiu CS, Crock LW, Morales ME, Ness TJ, Gereau RW 4th. Activation of spinal extracellular signal-regulated kinases (ERK) $1 / 2$ is associated with the development of visceral hyperalgesia of the bladder. Pain 2011;152:2117-24.

28. Johansen C, Verheul A, Gram L, Gill T, Abee T. Protamine-induced permeabilization of cell envelopes of gram-positive and gram-negative bacteria. Appl Environ Microbiol 1997;63:1155-9.

29. Yoshikawa S, Kawamorita N, Oguchi T, Funahashi Y, Tyagi P, Chancellor $\mathrm{MB}$, et al. Pelvic organ cross-sensitization to enhance bladder and urethral pain behaviors in rats with experimental colitis. Neuroscience 2015;284:422-9.

30. Luber-Narod J, Austin-Ritchie T, Banner B, Hollins C 3rd, Maramag $\mathrm{C}$, Price $\mathrm{H}$, et al. Experimental autoimmune cystitis in the Lewis rat: a potential animal model for interstitial cystitis. Urol Res 1996;24:367-73.

31. Lin YH, Liu G, Kavran M, Altuntas CZ, Gasbarro G, Tuohy VK, et al. Lower urinary tract phenotype of experimental autoimmune cystitis in mouse: a potential animal model for interstitial cystitis. BJU Int 2008;102:1724-30.

32. Marson L, Giamberardino MA, Costantini R, Czakanski P, Wesselmann U. Animal models for the study of female sexual dysfunction. Sex Med Rev 2013;1:108-22.

33. Fariello JY, Moldwin RM. Similarities between interstitial cystitis/ bladder pain syndrome and vulvodynia: implications for patient management. Transl Androl Urol 2015;4:643-52.

34. Rudick CN, Schaeffer AJ, Klumpp DJ. Pharmacologic attenuation of pelvic pain in a murine model of interstitial cystitis. BMC Urol 2009;9:16.

35. Yang W, Yaggie RE, Jiang MC, Rudick CN, Done JD, Heckman CJ, et al. Acyloxyacyl hydrolase modulates pelvic pain severity. Am J Physiol Regul Integr Comp Physiol 2017 Nov 8:ajpregu.00239.2017 [Epub]. https://doi.org/10.1152/ajpregu.00239.2017.

36. Sadler KE, Stratton JM, Kolber BJ. Urinary bladder distention evoked visceromotor responses as a model for bladder pain in mice. J Vis Exp 2014 Apr 27;(86). https://doi.org/10.3791/51413.

37. Wang X, Liu W, O’Donnell M, Lutgendorf S, Bradley C, Schrepf A, et al. Evidence for the role of mast cells in cystitis-associated lower urinary tract dysfunction: a multidisciplinary approach to the study of chronic pelvic pain research network animal model study. PLoS 
One 2016;11:e0168772.

38. Powell N, Walker MM, Talley NJ. The mucosal immune system: master regulator of bidirectional gut-brain communications. Nat Rev Gastroenterol Hepatol 2017;14:143-59.

39. Hunter HJ, Momen SE, Kleyn CE. The impact of psychosocial stress on healthy skin. Clin Exp Dermatol 2015;40:540-6.

40. Leue C, Kruimel J, Vrijens D, Masclee A, van Os J, van Koeveringe G. Functional urological disorders: a sensitized defence response in the bladder-gut-brain axis. Nat Rev Urol 2017;14:153-63.

41. Lee UJ, Ackerman AL, Wu A, Zhang R, Leung J, Bradesi S, et al. Chronic psychological stress in high-anxiety rats induces sustained bladder hyperalgesia. Physiol Behav 2015;139:541-8.

42. Smith AL, Leung J, Kun S, Zhang R, Karagiannides I, Raz S, et al. The effects of acute and chronic psychological stress on bladder function in a rodent model. Urology 2011;78:967.e1-7.

43. Wang Z, Chang HH, Gao Y, Zhang R, Guo Y, Holschneider DP, et al. Effects of water avoidance stress on peripheral and central responses during bladder filling in the rat: a multidisciplinary approach to the study of urologic chronic pelvic pain syndrome (MAPP) research network study. PLoS One 2017;12:e0182976.

44. Westropp JL, Buffington CA. In vivo models of interstitial cystitis. J Urol 2002;167(2 Pt 1):694-702.

45. Buffington CA, Pacak K. Increased plasma norepinephrine concentration in cats with interstitial cystitis. J Urol 2001;165(6 Pt 1):20514.

46. Matos R, Serrão P, Rodriguez L, Birder LA, Cruz F, Charrua A. The water avoidance stress induces bladder pain due to a prolonged alpha1A adrenoceptor stimulation. Naunyn Schmiedebergs Arch Pharmacol 2017;390:839-44.

47. Charrua A, Pinto R, Taylor A, Canelas A, Ribeiro-da-Silva A, Cruz $\mathrm{CD}$, et al. Can the adrenergic system be implicated in the pathophysiology of bladder pain syndrome/interstitial cystitis? A clinical and experimental study. Neurourol Urodyn 2015;34:489-96.
48. Matos R, Cordeiro JM, Coelho A, Ferreira S, Silva C, Igawa Y, et al. Bladder pain induced by prolonged peripheral alpha $1 \mathrm{~A}$ adrenoceptor stimulation involves the enhancement of transient receptor potential vanilloid 1 activity and an increase of urothelial adenosine triphosphate release. Acta Physiol (Oxf) 2016;218:265-75.

49. Clemens JQ, Mullins C, Kusek JW, Kirkali Z, Mayer EA, Rodríguez $\mathrm{LV}$, et al. The MAPP research network: a novel study of urologic chronic pelvic pain syndromes. BMC Urol 2014;14:57.

50. Lai H, Gereau RW 4th, Luo Y, O’Donnell M, Rudick CN, Pontari M, et al. Animal models of urologic chronic pelvic pain syndromes: findings from the multidisciplinary approach to the study of chronic pelvic pain research network. Urology 2015;85:1454-65.

51. Elbadawi A. Interstitial cystitis: a critique of current concepts with a new proposal for pathologic diagnosis and pathogenesis. Urology 1997;49(5A Suppl):14-40.

52. Tomaszewski JE, Landis JR, Russack V, Williams TM, Wang LP, Hardy C, et al. Biopsy features are associated with primary symptoms in interstitial cystitis: results from the interstitial cystitis database study. Urology 2001;57(6 Suppl 1):67-81.

53. Leiby BE, Landis JR, Propert KJ, Tomaszewski JE; Interstitial Cystitis Data Base Study Group. Discovery of morphological subgroups that correlate with severity of symptoms in interstitial cystitis: a proposed biopsy classification system. J Urol 2007;177:142-8.

54. Sant GR, Kempuraj D, Marchand JE, Theoharides TC. The mast cell in interstitial cystitis: role in pathophysiology and pathogenesis. Urology 2007;69(4 Suppl):34-40.

55. Logadottir Y, Delbro D, Lindholm C, Fall M, Peeker R. Inflammation characteristics in bladder pain syndrome ESSIC type 3C/classic interstitial cystitis. Int J Urol 2014;21 Suppl 1:75-8.

56. van de Merwe JP, Nordling J, Bouchelouche P, Bouchelouche K, Cervigni M, Daha LK, et al. Diagnostic criteria, classification, and nomenclature for painful bladder syndrome/interstitial cystitis: an ESSIC proposal. Eur Urol 2008;53:60-7. 